Cahiers québécois de démographie

Fichiers généalogiques et analyse de la structure génétique de populations non isolées. Application à une population agricole du Limousin

GENEALOGICAL FILES AND THE ANALYSIS OF THE GENETIC STRUCTURE OF NON-ISOLATED POPULATIONS. AN APPLICATION TO A RURAL POPULATION OF THE LIMOUSIN REGION

REGISTROS GENEALOGICOS Y ANALISIS DE LA ESTRUCTURA GENETICA DE POBLACIONES NO APARTADAS. APLICACION A UNA POBLACION AGRICOLA DE LA REGION DEL LIMOUSIN

André Sevin et Gilles Boetsch

Volume 20, numéro 1, printemps 1991

URI : https://id.erudit.org/iderudit/010064ar

DOI : https://doi.org/10.7202/010064ar

Aller au sommaire du numéro

Éditeur(s)

Association des démographes du Québec

ISSN

0380-1721 (imprimé)

1705-1495 (numérique)

Découvrir la revue

\section{Citer cet article}

Sevin, A. \& Boetsch, G. (1991). Fichiers généalogiques et analyse de la structure génétique de populations non isolées. Application à une population agricole du Limousin. Cahiers québécois de démographie, 20(1), 37-50.

https://doi.org/10.7202/010064ar
Résumé de l'article

Cet article montre les problèmes méthodologiques qui se posent lors de l'élaboration de fichiers généalogiques pour des populations peu endogames ou « ouvertes ». L'aire d'étude doit être étendue à un espace géographique plus vaste que celui dans lequel réside la population retenue.
Tous droits réservés @ Association des démographes du Québec, 1991
Ce document est protégé par la loi sur le droit d'auteur. L'utilisation des services d'Érudit (y compris la reproduction) est assujettie à sa politique d'utilisation que vous pouvez consulter en ligne.

https://apropos.erudit.org/fr/usagers/politique-dutilisation/ 
Cahiers québécois de démographie

Vol. 20, no 1, printemps 1991, p. 37-50

\title{
Fichiers généalogiques et analyse de la structure génétique de populations non isolées. Application à une population agricole du Limousin
}

\author{
André SEVIN et Gilles BOETSCH *
}

La connaissance des phénomènes démographiques, en particulier de ceux quil touchent aux mécanismes de la reproduction, est essentielle pour suivre l'évolution du pool génique des populations humaines. Les méthodes qu'elle nécessite dêpendent essentiellement de deux facteurs : la dimension de l'unité de population étudiée et la nature de l'information dont on dispose.

Le développement des études démographiques en anthropologie est directement lié au succès de la génétique des populations (Baker et Sanders, 1973); ces études ont nécessité la mise en place de méthodologies adaptées à la spécificité de l'objet d'étude : des petits groupes humains souvent isolés de leurs voisins (Jakobi, 1984; Howell, 1986).

Cela explique pourquol, jusqu'à présent, les travaux des gênéticiens et des anthropobiologistes intéressés aux populations humaines ont pour la plupart porté sur des populations explicitement définies, les isolats, isolées par des barrières géographiques (voir en particulier Roberts, 1967; Benoist, 1964) ou culturelles (voir entre autres Bonné, 1963, Chapman et Jacquard, 1971; Chaventré, 1971; Howell, 1979; Cazes, 1986).

Parmi les travaux concernant les structures des populations humaines, certains se sont appuyés sur des informations recueillies oralement, d'autres sur des sources écrites, c'est-àdire principalement sur des archives d'êtat civil. C'est le cas de

Respectivement du Centre national de la recherche scientifique, Centre hospitalier universitaire Purpan, Toulouse, et du Centre national de la recherche scientifique, Université d'Aix-Marseille III, Aix-en-Provence. 
très nombreuses études : citons celles qui ont étè effectuées dans les vallées pyrénéennes françaises, sur la vallée de l'Ouzom (Serre et al., 1985), le pays de Sault (Vu Tien Khang et Sevin, 1977) ou le Lavedan (Guy, 1988); celles qui ont été faites sur les Alpes suisses (notamment Morton et al., 1968; Hussels, 1969; Friedl et Ellis, 1974; Ellis et Stramer, 1978); ou encore celles qui portent sur des communautés rurales canadiennes (par exemple Gomila et Guyon, 1969; Philippe et Gomila, 1971; Mayer, 1981; Bouchard, 1984).

Ces aires d'études étaient très endogames et il fut relativement aisé de reconstituer de bonnes généalogies familiales; l'histoire génétique ou sociale que l'on a pu écrire n'a souffert que de légers manques, dus essentiellement aux "trous" que présentait l'information écrite.

Dans le cas de populations sans archives écrites, où les reconstitutions risquent d'être peu profondes, les données hématologiques (systèmes érythrocytaires, enzymatiques, immunoglobulines, protêines sériques, hémoglobines, système HL-A et son complément) procurent une image de la structure génétique de la population à un instant donné. Tel est le cas des Yanomama (Ward et al., 1975), des Semaï (Fix, 1982), de la population d'Idélès (Lefèvre-Witier, 1982).

Lorsque les individus sont bien identifiés, comme dans la population Touareg Kel Kummer (Chaventré, 1983), les données généalogiques vont se situer dans une perspective d'analyse puis de compréhension des mécanismes de transmission et d'évolution des patrimoines génétiques.

\section{PROBLEMATIgUE}

L'utilisation de l'informatique dans l'étude pionnière de Mange (1964a) sur l'évolution de la structure gẻnétique des Huttérites a permis le développement de travaux portant sur des populations de plus en plus vastes (voir par exemple Skolnick et al., 1984, et Bouchard, 1984). Les méthodes de gestion de fichiers de populations et les programmes de reconstitutions généalogiques sont devenus de plus en plus sophistiqués (Sutter et Tabah, 1956; Mange, 1964b; Mugnier et al., 1966; MacKusick, 1967; Edwards, 1967; Hilden, 1967; MacLean, 1969; Cuisenier et al., 1970; Elston, 1970; Skolnick et al., 1971; Garçon et Jacquard, 1971; Beauchamp et al., 1973; Gutman, 1977; Pelissier, 1983; Ryan, 1985; etc.). 
Cet accroissement des moyens de gestion, de stockage et d'analyse des fichiers a permis de passer à des études concernant des populations non seulement de plus en plus vastes, mais aussi de plus en plus complexes. Tel est le cas des populations dites "ouvertes" (et non forcément exogames), c'est-àdire celles dont une grande partie des membres se marient avec des individus de communautés voisines, selon des règles matrimoniales qui mettent en jeu un certain nombre de critères et non un seul (la parenté). Lors de l'étude des caractères culturels ou sociaux qui peuvent se substituer à des barrières géographiques et donner un sentiment d'identité à la communauté, il convient aussi de trouver les facteurs qui agissent dans le sens de l'éclatement ou du fractionnement de la population en sousunités tendant à devenir semi-perméables voire imperméables les unes aux autres. Suivant les lieux et les époques, certains facteurs pourront tour à tour favoriser l'endogamie dans un groupe et l'exogamie dans un autre. Si, dans certaines communautês, le facteur religieux peut se rêvéler discriminant, dans d'autres, c'est la contrainte imposée par le mode de dévolution des biens qui devient l'agent principal du comportement matrimonial.

Dans la région limousine ${ }^{1}$ que nous étudions, le mode de dévolution des biens est assurément un facteur contraignant. C'est à partir de sa transcription matérielle dans l'exploitation agricole que nous avons tenté de définir une aire géographique pertinente au sein de laquelle s'exerce toute une sêrie de pratiques sociales, dont bien sûr le choix du conjoint.

La particularité de l'habitat traditionnel marque la mentalité paysanne limousine : il consiste en un groupement de quelques fermes (trois à six) dans une unité structurelle dénommée "hameau". Cet habitat, spécifique des pays de petites montagnes, a permis une appropriation fonctionnelle de ce type d'espace rural et, par là, a généré une aire matrimoniale souvent structurée par un espace généalogique. La détermination de cet espace passe, pour le chercheur, par la reconnaissance d'une succession de niveaux géo-administratifs comme la commune, le canton ou le département, niveaux population-

1 Il s'agit du canton de Châteauponsac, unité administrative regroupant cinq communes. Il comprend 9000 habitants et fait partie d'une zone rurale dénommée "Marche limousine. Située dans le Nord de la région limousine, cette zone rurale se caractérise par des petites exploitations agricoles, en mode de faire-valoir direct avec l'élevage bovin comme activité agricole dominante. 
nels de plus en plus vastes dans lesquels la probabilité de trouver un conjoint serait de plus en plus faible (il existe plus de chances de trouver un conjoint dans le canton de Châteauponsac, qui compte 9000 habitants, que dans le reste du département, qui ccmpte 300000 habitants). Cela nous renvoie au modèle d'isolation par distance (Wright, 1946), c'est-à-dire à la faible probabilité qui existe pour un individu de rencontrer un conjoint habitant une région éloignée de la sienne.

C'est au cours du dépouillement de l'enquête et des premières analyses que cette aire matrimoniale s'est peu à peu révélée (Crognier et al., 1984; Boetsch et Sevin, 1988) : elle a comme caractéristique de déborder l'unité communale et de s'accroitre en fonction du temps.

Une des finalités de ce genre d'étude est de définir la limite du cercle endogame avec une certaine précision. L'accroissement du nombre de conjoints "exogames" au cours du temps augmente le nombre de chaînes gênéalogiques "extérieures", car plus on étend le périmètre d'investigation, plus on retrouve de "fondateurs", mais plus on cerne l'espace généalogique réel de la population. Cette préoccupation doit être sans cesse présente car de trop nombreuses études ont mal embrassé la véritable dimension de la population en travaillant sur des échelles spatiales trop petites (Bates et Lees, 1979).

À l'opposé, les études sur des populations apparemment très "ouvertes", comme cela peut être le cas dans les tissus suburbains en formation rapide, sont extrêmement complexes à mener car le cercle des unions est lâche, donc très peu dêlimité. Il convient donc de déterminer un coefficient dit de "migration" correspondant au rapport nombre de fondateurs/nombre d'ancêtres. Si la région était complètement endogame, ce rapport serait toujours nul (isolat), mais cette configuration ne se rencontre jamais dans la réalité. Lorsque ce coefficient s'accroît très vite, il devient délicat d'accorder trop de pertinence à l'étude entreprise. En effet, ce cas de figure correspond à une population non isolée qui renouvelle son pool de "fondateurs" à chaque génération. Mais le cas fréquemment rencontré est celui de populations traditionnellement endogames qui, à une période particulière et pour des raisons économiques variées (marchés attractifs, industries ou thermalisme), voient arriver parmi elles un certain nombre de "migrants".

L'espace généalogique de cette population est assez bien cerné jusqu'à la septième génération, puisque moins de $30 \%$ des ancêtres ne sont pas identifiés. Mais ces personnes 
"inconnues" (fondateurs) ne sont pas forcément des migrants extérieurs à la petite région : il s'agit souvent d'individus difficilement identifiables dans les actes anciens de l'êtat civil (omissions d'actes, faiblesse du nombre de patronymes, faible variabilité des prénoms).

\section{APPLICATION À UNE POPULATION AGRICOLE FRANÇAISE}

À partir d'une fraction de la population agricole du canton de Châteauponsac (Haute-Vienne), nous avons reconstruit un faisceau de chaînes généalogiques. Dans un premier temps, nous avons reconstitué toutes les fratries des individus ancêtres de notre population et ayant vécu dans le canton depuis le début du XIXe siècle. Nous avons enregistré ces fratries sous la forme de fiches dites "de Henry" (révisées par Mugnier et al., 1966) adaptées à notre traitement en codant au maximum l'information qui nous était utile.

Par exemple, dès que les ancêtres sortent du canton ou encore ont vécu au XVIIIe siècle, nous ne prenons en compte que l'information minimale sur les fratries, c'est-à-dire les enfants utiles avec leurs liens de parenté.

La reconstitution est basée sur la règle des triplets : tout enfant d'une fratrie possède un numéro unique qui le caractérise et deux parents toujours définis eux-mêmes par leur numéro d'enfant dans leur propre fratrie. Seuls les "fondateurs" sont considérés comme la dernière information sur l'ancêtre. Tous les couples parentaux étaient repérés par un numéro de fratrie.

Nous avons donc constitué quatre fichiers : F1, F2, F3 et F4 (figure 1) : F1 et F2 sont les fichiers de familles du canton; F3 et F4 sont les fichiers de familles vivant hors du canton; F1 et F3 représentent la période des XIXe et XXe siècles; F2 et F4 représentent le XVIIIe siècle.

Pour vérifier la validité de notre échantillon, il fallait voir si nous remontions bien dans les généalogies (tableau 1). Pour une profondeur de neuf générations, nous avons pu identifier $54 \%$ des ancêtres chez les hommes et $33 \%$ chez les épouses. Cela correspond en moyenne respectivement à sept et à cinq génêrations.

\section{CREATION DES FICHIERS GENEALOGIGUES}

La première étape consiste à transcrire les informations recueillies sur des bordereaux de type "fiche de Henry". Nous 


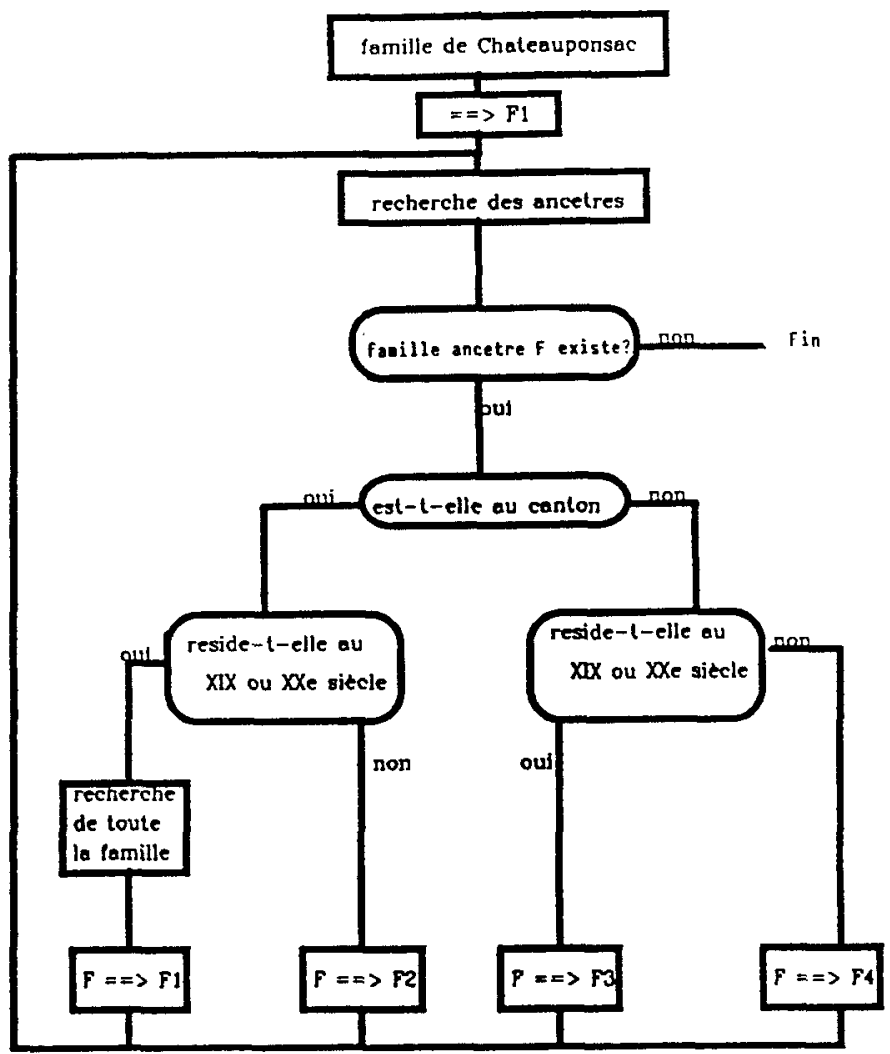

Figure 1 - ORGANIGRAMME DE LA CONSTRUCTION DES FICHIERS

TABLEAU 1

Profondeur d'information pour les 97 familles actuelles

\begin{tabular}{ccccccccccc}
\hline $\begin{array}{c}\text { LONGUEUR LIGNÉE } \\
\text { Femmes }\end{array}$ & $\rightarrow$ & 2 & 3 & 4 & 5 & 6 & 7 & 8 & 9 & Total \\
Hommes & $\downarrow$ & & & & & & & & & \\
1 & 6 & & & 1 & & & & 1 & 1 & 9 \\
2 & 1 & & 1 & & & & & 3 & & 5 \\
3 & 1 & & & & 1 & & & 1 & & 3 \\
4 & 2 & & & & & & 1 & & & 3 \\
5 & & & & & & & & & & 0 \\
6 & 2 & 2 & 1 & 1 & & & 1 & & & 3 \\
7 & 5 & 3 & 1 & 1 & 1 & & 5 & 11 & 6 & 33 \\
8 & 4 & 5 & 1 & 1 & 1 & 1 & 2 & 4 & 3 & 22 \\
9 & 21 & 10 & 5 & 4 & 3 & 5 & 12 & 27 & 10 & 97 \\
Total & & & & & & & & & & \\
\hline
\end{tabular}


avons essayé de regrouper les patronymes en classes définies par cinq lettres (396 patronymes différents sur un total de 1973 dans le fichier des familles ayant vécu dans le canton de Châteauponsac au XIXe et au XXe siècle). Le plan d'enregistrement est le suivant pour chaque famille.

Pour chaque enfant d'une famille, deux zones d'identification sont créées.

- Une zone d'identification de ego

- un numéro de fratrie

- un numéro d'individu

- le sexe de l'individu

- sa date de naissance

- son lieu de naissance

- sa date de décès
- son lieu de décès

- un code état matrimonial au moment du décès

- un code de profession au décès

- un code "hëritier".

- Une zone concernant les parents de l'individu commune à chaque enfant de la fratrie

- pour chaque conjoint :

- un numéro

d'identification

- un numéro d'ordre de mariage

- un code profession

- un lieu de résidence avant le mariage

- une date de mariage
- un lieu de mariage

- un lieu de résidence après le mariage

- les deux patronymes en abrégé

- le nombre d'enfants procréés

- la profession du mari à la naissance de l'enfant.

Une fois les familles reconstituées, nous avons essayé de rechercher le maximum d'informations généalogiques à partir des couples parentaux. Nous avons pu ainsi retrouver de nombreuses connexions parentales malgré le fait qu'un certain nombre d'ancêtres sortent du cadre cantonal. Cette manière de procéder nous permettait de préserver l'information "consanguinité" venant de l'extérieur. Seule a pu échapper aux mailles de l'enquête une consanguinité provenant de personnes très éloignées de la région et surtout non notées dans les registres. Cela représente une perte d'information extrêmement faible, comme nous le verrons plus loin.

Ainsi avons-nous créé une "fiche familiale" pour tout individu participant aux lignées gênéalogiques à partir du moment où nous avions des renseignements concernant au moins un de ses parents. Lorsque l'information s'arrête, nous sommes en 
présence de "fondateurs" pour lesquels nous ne possédons qu'une origine géographique et une date de naissance plus ou moins précise.

La reconstruction de lignées généalogiques ascendantes est associée à l'information obtenue dans les actes de l'état civil. En retour, les éléments d'information démographique rencontrés après les reconstructions généalogiques retracent la dynamique des tendances démographiques des individus "utiles" des diverses générations (Bibeau et Poulain, 1984). Lorsque l'on reconstitue les caractéristiques démographiques élémentaires (naissances, mariages, décès) des couples d'ancêtres, on constate que les actes de mariage demeurent les élêments les plus aisément identifiables au cours du temps. Les actes de naissance des individus sont facilement identifiables à partir de 1793. Par contre, l'information concernant les actes de décès est faible dans le canton avant 1793; elle est encore plus rare pour les individus ayant résidê hors du canton (tableau 2).

TABLEAU 2

Tableau 1 regroupé

\begin{tabular}{lcccc}
\hline Femmes & $\begin{array}{c}\text { Peu } \\
\text { d'information } \\
\text { <4 générations }\end{array}$ & $\begin{array}{c}\text { Information } \\
\text { moyenne } \\
\text { 4-6 génér. }\end{array}$ & $\begin{array}{c}\text { Bonne } \\
\text { information } \\
>6 \text { générations }\end{array}$ & Total \\
\hline Hommes & & & & \\
< 4 générations & 9 & 2 & 6 & 17 \\
4-6 générations & 3 & 1 & 2 & 6 \\
$>$ 6 générations & 24 & 9 & 41 & 74 \\
\hline Total & 36 & 12 & 49 & 97 \\
\hline
\end{tabular}

Le fichier généalogique comprend en fait deux types d'enregistrement :

- un premier enregistrement de type "familial", où les gens sont numérotês et regroupés en familles et où chaque individu est associé aux numéros de ses deux parents. Nous avons adopté un code spécial pour reconnaître les fratries entièrement reconstituées des familles incomplètes;

- un second enregistrement dit "de fondateurs", pour lequel nous ne possédons plus l'information sur les parents (codés 00000). 
Dans l'analyse génétique, c'est l'ensemble de l'information utile et seulement cette information qui est conservée. Elle est résumée en deux chiffres pour l'étude de notre échantillon de Châteauponsac : 2881 "enfants utiles" (c'est-à-dire 2881 triplets) et 1892 "fondateurs" (soit 1892 origines géographiques). C'est à partir de ces informations que nous avons étudié la probalité d'origine des gènes et la consanguinité.

Par exemple, nous avons calculé, pour chaque famille, la probabilité d'origine des gènes en créant un "vecteur ligne" débutant par les numéros des deux conjoints suivis des numéros de leurs parents, puis de leurs grands-parents, etc., jusqu'à ce que le pointeur nous indique que nous n'avions plus d'information sur la lignée familiale. Nous étions alors parvenus au niveau des "fondateurs". Nous avons ensuite parcouru ce vecteur en ignorant les individus "non fondateurs" et en remplaçant les numéros de fondateurs par leur code d'origine géographique. Dans ce vecteur ainsi "corrigê", on ne rencontre que des indices correspondant à des générations, des "origines de fondateurs". Pour chaque famille, nous pouvons ainsi calculer la contribution exacte des communes avoisinantes (à la génération $x$ où $1 l$ apparaît, un village apporte $(1 / 2) \times$ pour la lignée). Nous pouvons voir comment a évolué le pool génique de notre population au cours des différentes périodes considérées.

\section{CONCLUSION}

Le problème posé à l'anthropologue comme à l'historien ou au démographe est de percevoir l'unité populationnelle pertinente lorsqu'il se propose d'étudier une population qualifiée d'nouverten, c'est-à-dire pratiquant un mariage exogame. On voit ici l'intérêt méthodologique de situer les enquêtes généalogiques dans une aire géographique plus vaste que celle où réside la population étudiée; cela permet de prendre en compte à la fois les variations spatiales et les variations temporelles des phénomènes, comme par exemple de retrouver une consanguinité qui peut être élevée dans la fraction des lignées "exogamiques", c'est-à-dire celles ayant vécu hors du canton (tableau 3).

D'autre part, lorsque nous regardons la relation qui existe entre le coefficient d'apparentement et l'origine géographique des conjoints, nous observons que l'apparentement est très élevé lorsque les époux sont originaires du même hameau, huit fois moins fort s'ils viennent de deux hameaux de la même 
TABLEAU 3

Part d'information démographique connue dans les gênérations ascendantes (\%)

\begin{tabular}{lcccccc}
\hline & & Canton & \multicolumn{3}{c}{ Hors canton } \\
& Naissance & Mariage & Décès & Naissance & Mariage & Décès \\
\hline 1720 & 11 & 66 & 47 & & & \\
1740 & 4 & 91 & 48 & 7 & 93 & 40 \\
1760 & 42 & 91 & 52 & 45 & 85 & 38 \\
1780 & 74 & 97 & 52 & 86 & 96 & 32 \\
1800 & 91 & 82 & 27 & 93 & 93 & 33 \\
1820 & 97 & 96 & 93 & 95 & 41 & 38 \\
1840 & 99 & 94 & 73 & 93 & 93 & 28 \\
1860 & 100 & 98 & 80 & 95 & 91 & 18 \\
1880 & 100 & 100 & 77 & 99 & 100 & 46 \\
1900 & 100 & 100 & 94 & 100 & 96 & 93 \\
1920 & 100 & 100 & 99 & 98 & 100 & 94 \\
1940 & 100 & 100 & 100 & 100 & 100 & 100 \\
\hline
\end{tabular}

commune et seize fois moins s'ils viennent de deux communes différentes. Cela montre que le hameau est une unité populationnelle extrêmement pertinente, ce qui ne serait pas le cas de la commune : on n'a que deux fois plus de chances de s'épouser dans la parenté si on se marie avec une personne de sa commune qu'avec une personne de la commune voisine. Lorsque le conjoint est originaire de plus de $25 \mathrm{~km}$, l'apparentement semble inexistant. Par contre apparait un mouvement faible, mais régulier, d'apport de couples "migrants" apparentés (surtout des métayers) originaires de communes voisines. Il serait sans doute très intéressant de conforter ce travail par une étude de l'évolution des usages locaux dans la région ainsi que par un dépouillement des actes notariés. Ce dernier volet donnerait une indication sur l'évolution du patrimoine familial et expliquerait certaines stratégies d'alliance.

L'étude des populations rurales vivant dans un enchevêtrement de réseaux d'alliance nécessite des fichiers gẻnéalogiques importants parce qu'ils doivent couvrir une aire géographique supérieure à celle de la communauté que l'on se propose d'étudier. Le fichier généalogique doit être spécifique, bien adapté à l'objet d'étude. Ainsi, les êtudes portant sur des populations urbaines à l'intérieur desquelles des communautés culturelles et sociales coexistent nécessiteront des fichiers de population d'une autre nature dans la mesure où lls devront intégrer les composants les plus structurants de ces communautés. 


\section{RÉFERENCES BIBLIOGRAPHIUES}

BAKER, P. T., et W. T. SANDERS, 1973. «Demographic Studies in Anthropology", Annual Review of Anthropology, 1, 151-178.

BATES, G., et S. H. LEES, 1979. "The Myth of Population Regulation*. In N. CHAGNON et W. IRON, éd. Evolutionary Biology and Social Behavior: An Anthropological Perspective. North Sciutate, Duxbury Press, 273-289.

BEAUCHAMP, P., H. CHARBONNEAU et Y. LAVOIE, 1973. "Reconstitution automatique des familles par le programme "Hochela" . Population, 28, 1, 39-58.

BENOIST, J., 1964. «Saint-Barthelemy: Physical Anthropology of an Isolate", American Journal of Physical Anthropology, 22, 473-488.

BIBEAU, A., et M. POULAIN, 1984. .De la généalogie à la démographie historique : généalogie ascendante et analyse démographique», Annales de démographie historique, 55-69.

BOETSCH, G., et A. SEVIN, 1988. «Inbreeding in a French Rural Population", International Journal of Anthropology, 4, 1-2, 27-37.

BONNÉ, B., 1963. "The Samaritans: A Demographic Study". Human Biology, 35, 61-89.

BOUCHARD, G., 1984. «Nouvelles perspectives pour les recherches génétiques. Le fichier-réseau de la population du Saguenay", Annales de démographie historique, 81-88.

CAZES, M. H., 1986. "Genetic Origins of the Dogon Population in the Arrondissement of Boni (Mali)». American Journal of Human. Genetics, 39, 96-111.

CHAPMAN, A. M., et A. JACQUARD, 1971. "Un isolat d'Amérique Centrale : les indiens Jicaques du Honduras*. In Génétique et population. Paris, INED, Cahier numéro 60, 163-185.

CHAVENTRÉ, A., 1971. „Présentation d'un isolat Targuix. In Génétique et population. Paris, INED, Cahier numéro 60, 187-199.

CHAVENTRÉ, A., 1983. Évolution anthropobiologique d'une population touarègue. Les Kel Kummer et leurs apparentés. Paris, INED, PUF.

CROGNIER, E., D. BLEY et G. BOETSCH, 1984. Mariage en Limousin. Paris, Éd. du CNRS.

CUISENIER, J., M. SEGALEN et M. VIRVILLE, 1970. Pour l'étude de la parenté dans les sociétés européennes : le programme d'ordinateur Archiv, L'Homme, X, 3, 27-74.

EDWARDS, A. W. F., 1967. *Automatic Construction of Genealogies from Phenotipic Information", Bulletin of the European Society of Human Genetics, 1, 42-43.

ELLIS, W., et W. STRAMER, 1978. «Inbreeding as Measured by Isonymy and by Pedigrees and Population Size in Törbel, Switzerland,. American Journal of Human Genetics, 30, 366-376.

ELSTON, R. C., et J. STEWART, 1971. *A General Model for the Analysis of Pedigree Datan, Human Heredity, 21, 523-542. 
FIX, A. G., 1982. "Genetic Structure of the Semai". In M. H. CRAWFORD et J. H. MEILKE, éd. Current Development in Anthropological Genetics. Vol. 2: Ecology and Population Structure. New York et Londres, Plenum Press, 179-204.

FRIEDL, J., et W. ELLIS, 1974. «Inbreeding, Isonymy and Isolation in a Swiss Communityn, Human Biology, 46, 4, 699-712.

GARÇON, C., et A. JACQUARD, 1971. „Programme de calcul des structures génétiques et des coefficients de consanguinité.. In Génétique et population. Paris, INED, Cahier numéro 60, 163-185.

GOMILA, J., et L. GUYON, 1969. «Étude comparative de deux petites communautês rurales*, Population, 24, 6, $1127-1153$.

GUTMAN, M. P., 1977. «Reconstituting Wandre. An Approach to Semiautomatic Family Reconstitution*, Annales de démographie historique, 315-341.

GUY, Y., 1988. Saint-Savin, démographie d'un village bigourdan 1618 1975. Paris, Éd. du CNRS.

HILDEN, J., 1967. "Formalized and Automated Pedigree Processing”, Bulletin of the European Society of Human Genetics, 1, 41.

HOWELL, N., 1979. Demography of the Dobel Kung. New York. Academic Press.

HOWELL, N., 1986. "Demographic Anthropology», Annual Review of Anthropology, 15, 219-246.

HUSSELS, I., 1969. "Genetic Structure of Saas, a Swiss Isolate", Human Biology, 41, 4, 469-479.

JAKOBI, L., 1984. "Les petits groupes humains : étude anthropologique et de génétique des populations". Bulletins et mémoires de la société d'anthropologie de Paris, série XIV, 1, 3, 225-236.

LEFÈVRE-WITIER, Ph., 1982. Idélès (Ahggar, Algérie) : structures et dynamique génétiques d'une communauté rurale du Sahara central. Toulouse, Thèse d'État en biologie humaine.

McKUSICK, V. A., 1967. „Genealogic and Bibliographic Applications of Computers in Human Genetics". Proceedings of the 3rd International Congress of Human Genetics, 483-488.

MACLEAN, C., 1969. "Computer Analysis of Pedigree Data". In N. E. NORTON, éd. Computer Applications in Genetics. Honolulu, University of Hawai Press, 82-86.

MANGE, A. P., 1964a. "Fortran Programs for Computing Wright's Coefficient of Inbreeding in Human and Non-human Populationsw. American Journal of Human Genetics, 16, 484.

MANGE, A. P., 1964b. "Growth and Inbreeding of a Human Isolate", Human Biology. 36, 2, 105-133.

MAYER, F., 1981. "Histoire démographique et destin biologique de deux villages québécoisn, Anthropologie et sociétés, 5, 2, 17-68.

MORTON, N., N. YASUDA, C. MIKI et S. YEE, 1968. "Population Structure of the ABO Blood Groups in Switzerland, American Joumal of Human Genetics, 25, 347-361. 
MUGNIER, M., M. SUTTER et J. M. GOUX, 1966. «Organigrammes pour l'étude mécanographique de la parenté et de la fécondité dans une population", Population, 21, 1, 75-98.

PELISSIER, J. P., 1983. Démographie, généalogie, micro-informatique. Paris, 2e Cahier des Annales de démographie historique.

PHILIPPE, P.. et J. GOMILA, 1971. «Stucture de population et mariages consanguins à l'Isle-aux-Coudres (Québec)», Population, 26, 4, 707717.

ROBERTS, D. F., 1967. The Development of Inbreeding in an Island Population", Ciencia e Cultura, 19, 1, 78-84.

RYAN, N., 1985. “Giree: A System for Interactive Display and Manipulation of Genealogical Datan, B.I.C.A., 3, 6-12.

SERRE, J. L., L. JAKOBI et M. C. BABRON, 1985. "A Genetic Isolate in the French Pyrenees: Probabilities of Origin of Genes and Inbreeding", Journal of Biosoc. Sci., 17, 405-414.

SKOLNICK, M., A. MORONI, C. CANNINGS et L. L. CAVALLI-SFORZA, 1971. "The Reconstruction of Genealogies from Parish Registers". In F. R. DODSON, D. G. KENDALL et P. TAUTU, èd. Mathematics in the Archeological and Historical Sciences. Edinburg University Press, 319-344.

SKOLNICK, M., L. BEAN, G. MINEAU et L. JORDE, 1984. «Etudes démographiques et génétiques et bases de données généalogiques de l'Utahy, Annales de démographie historique, 103-114.

SUTTER, J., et L. TABAH, 1956. *Méthode mécanographique pour établir la généalogie d'une population. Applications à l'étude des esquimaux polaires", Population, 11, 507-530.

VU TIEN KHANG, J., et A. SEVIN, 1977. Choix du conjoint et patrimoine génétique. Étude de quatre villages du pays de Sault de 1740 à nos jours. Paris, Éd. du CNRS.

WARD, R. H., H. GERSHOWITZ, M. LAYRISSE et J. V. NEEL, 1975. *The Genetic Structure of a Tribal Population, the Yanomama Indians XI Gene Frequencies for 10 Blood Groups and the ABH-Le Secretor Traits in the Yanomama and their Neighbors", American Journal of Human Genetics, 27, 1-30.

WRIGHT, S., 1946. «Isolation by Distance under Diverse Systems of Mating", Genetics, 31, 39-59. 


\section{RESUME - SUMMARY - RESUMEN}

SEVIN André et Gilles BOETSCH - FICHIERS GENEALOGIQUES ET ANALYSE DE LA STRUCTURE GENETIQUE DE POPULATIONS NON ISOLEES. APPLICATION A UNE POPULATION AGRICOLE DU LIMOUSIN.

Cet article montre les problèmes méthodologiques qui se posent lors de l'élaboration de fichters généalogiques pour des populations peu endogames ou rouvertes". L'aitre d'étude doit être étendue à un espace géographique plus vaste que celui dans lequel réside la population retenue.

SEVIN André and Gilles BOETSCH - GENEALOGICAL FILES AND THE ANALYSIS OF THE GENETIC STRUCTURE OF NON-ISOLATED POPULATIONS. AN APPLICATION TO A RURAL POPULATION OF THE LIMOUSIN REGION.

This article shows the methodological problems appearing in the production of genealogical files for non-endogamous or "open" populations. The study area must be extended to a geographic space larger than the one where the designed population resides.

SEVIN André Y Gilles BOETSCH - REGISTROS GENEALOGICOS Y ANALISIS

DE LA ESTRUCTURA GENETICA DE POBLACIONES NO APARTADAS. APLICACION A UNA POBLACION AGRICOLA DE LA REGION DEL LIMOUSIN

Este articulo muestra los problemas que se plantean durante la elaboración de registros genealógicos para poblaciones poco endógamas, o "ablertas". Debe ampliarse el área de estudio a una zona geográfica más extensa que aquella en la cual reside la poblacion seleccionada. 